\title{
Association between angiotensin-converting enzyme insertion/deletion polymorphisms and intracranial aneurysm susceptibility: A meta-analysis
}

\author{
YAN-PING CUN ${ }^{1 *}$, CHENG-JIE XIONG $^{2 *}$, BO DIAO $^{3}$, YU YANG $^{4}$, LI PAN $^{3}$ and LIAN-TING MA ${ }^{1,3}$ \\ ${ }^{1}$ Postdoctoral Research Station of Neurosurgery and Departments of ${ }^{2}$ Orthopaedics, ${ }^{3}$ Neurosurgery and \\ ${ }^{4}$ Healthcare, Wuhan General Hospital of Guangzhou Military Command, Wuhan, Hubei 430070, P.R. China
}

Received June 6, 2016; Accepted September 19, 2016

DOI: $10.3892 /$ br.2017.893

\begin{abstract}
Various studies have evaluated the association between polymorphisms of angiotensin-converting enzyme (ACE) and intracranial aneurysm (IA) risk; however, the results remain inconsistent. The PubMed, Embase, and Wanfang Data databases were systematically searched until January 6th 2016. Case-control studies investigating the association between the ACE polymorphism and IA risk were included. Odds ratios (ORs) with $95 \%$ confidence intervals (CIs) were calculated with the fixed or random-effects model assuming allele, homozygote comparison of codominant, heterozygote comparison of codominant, dominant, and recessive models. Seven studies including 1,074 cases and 1,500 controls were included in the current meta-analysis. The results of the analysis indicated that the ACE polymorphism significantly increased IA risk in the allele, homozygote comparison of codominant and dominant models. According to the further stratified analysis by ethnicity, source of control and sample sizes, a significant association was identified between the ACE variant and IA risk in Asian individuals, hospital-based, or large (>300) subgroups in all of the genetic models, not including the recessive model. Furthermore, no significantly increased risk was indicated in Caucasian individuals, population-based, or small $(<300)$ subgroups in the heterozygote comparison of codominant, dominant and recessive models. The available evidence indicates that the ACE polymorphism is associated with an increased risk of IA, particularly in Asian individuals. However, other factors may impact this association. Further
\end{abstract}

Correspondence to: Professor Lian-Ting Ma, Postdoctoral Research Station of Neurosurgery, Wuhan General Hospital of Guangzhou Military Command, 627 Wuluo Road, Wuhan, Hubei 430070, P.R. China

E-mail:mlt1937@163.com

*Contributed equally

Key words: angiotensin-converting enzyme, intracranial aneurysm, genetics, polymorphism, meta-analysis large, well-designed multicenter studies are required to validate the findings from the present study.

\section{Introduction}

The incidence of intracranial aneurysm (IA) is high at approximately $2-3 \%$ in the general population worldwide (1). IA rupture is the most common reason for life-threatening subarachnoid hemorrhages (SAH), which accounts for $\sim 85 \%$ of overall SAH (2). Despite advances in diagnosis and treatment, the rates of mortality and morbidity of SAH associated with a ruptured IA remain high (3). Therefore, it is important to understand the molecular pathogenesis of IA to reduce the occurrence of SAH.

There is evidence to indicate that environmental and genetic factors are associated with the pathogenesis of IAs $(4,5)$. The modifiable factors, such as smoking, hypertension, and excessive alcohol consumption have been reported to lead to IA formation (6). In addition, accumulating evidence indicates that genetics are significant in the pathogenesis of IA. Familial IA is not rare, accounting for $7-20 \%$ of patients with aneurysmal SAH (7). The risk of experiencing unruptured IAs is significantly higher ( 4 times) in familial IA families than in the general population (8). Furthermore, certain genetic factors have been investigated and identified to be associated with IA (9-13), including angiotensin-converting enzyme (ACE).

$\mathrm{ACE}$ is the key enzyme in the renin-angiotensinaldosterone system (RAAS), as an important modulator of cerebrovascular disease (CVD) (14). The ACE gene, which consists of 26 exons and 25 introns is located on chromosome $17 q 23$ (14). The $A C E$ gene contains functional insertion/deletion (I/D) polymorphism of a 287-bp Alu sequence within intron 16 (15). Individuals with the DD genotype exhibit increased serum ACE levels and activity when compared with individual with the ID and II genotypes $(15,16)$. In addition, increased serum ACE levels may contribute to vascular injury, which is hypothesized to confer increased risk of IA.

Certain previous studies investigated the influence of $A C E$ I/D gene polymorphisms on IA susceptibility (17-23); however, the results of these studies remain inconsistent. As a single study might not be powered to demonstrate the overall effects, 
a meta-analysis using currently available data was performed in the present study to detect the potential association of ACE I/D gene polymorphisms on IA risk.

\section{Materials and methods}

Publication search. The search was performed using PubMed (https://www.ncbi.nlm.nih.gov/pubmed), Embase (www. embase.com), and Wanfang databases (updated to January 6th, 2016; http://www.wanfangdata.com.cn) with the following terms: 'Polymorphism', 'genotype', 'allele', 'mutation', 'variant', in combination with 'cerebral aneurysm', 'brain aneurysm', 'intracranial aneurysm', 'SAH', 'subarachnoid hemorrhage', in combination with 'ACE', 'angiotensin converting enzyme', and 'insertion/deletion'. The search was conducted without a limitation on language. Reference lists of eligible studies were also retrieved to find additional articles.

Inclusion criteria. Two reviewers independently screened the literature for relevance and disagreements were resolved by consensus. Studies included in the current meta-analysis met the following criteria: The studies i) evaluated the $A C E$ gene polymorphisms and IA risk; ii) were case-control studies; iii) contained available genotype frequencies for calculating odds ratios (ORs) with their $95 \%$ confidence interval (CI). The exclusion criteria were as follows: i) Duplicated studies; ii) limited sample size; iii) reviews, editorials or comments.

Data extraction. Information was carefully extracted from all eligible publications independently by two authors of the present paper. For conflicting evaluations, agreement was reached by discussion. For each study, the following characteristics were considered: First author surname, year of publication, country of origin, ethnicity of study subjects, source of control groups (population- or hospital-based controls), total number of cases and controls, genotyping method, evidence of Hardy-Weinberg equilibrium (HWE) and numbers of cases and controls with the II, ID, and DD genotypes for ACE. Subjects were categorized as East Asian and Caucasian.

Statistical analysis. Deviation from HWE was examined by $\chi^{2}$ test and $\mathrm{P}<0.05$ was considered to indicate a statistically significant difference. The strength of associations between $A C E$ gene polymorphisms and IA risk were measured by OR with 95\%CI. The pooled ORs were performed for I allele contrast (I vs. D), homozygote comparison of codominant, homozygote comparison of codominant (II vs. DD) and heterozygote comparison of codominant (ID vs. DD), dominant model (II+ID vs. DD), and recessive model (II vs. ID+DD), respectively. Heterogeneity assumption was verified by $\chi^{2}$-based Q-test and quantified using the $\mathrm{I}^{2}$ value. If the studies lacked heterogeneity $\left(P_{h}>0.1\right.$ and $\left.\mathrm{I}^{2}<50 \%\right)$, the fixed-effects model (the Mantel-Haenszel method) was adopted to calculate the overall ORs value (24). Otherwise, the random-effects model (the DerSimonian and Laird method) were used (25). To assess the stability of the results, sensitivity analyses were performed by removing each study individually, and recalculating the OR and the 95\% CI. Publication bias was assessed using Begg's funnel plots and Egger's linear regression test, and $\mathrm{P}<0.05$ was considered to indicate a statistically significant difference (26).
All statistical analyses were performed with Stata software (version 12.0; StataCorp LP, College Station, TX, USA), using two-sided P-values.

\section{Results}

Study characteristics. Our initial search identified 43 studies according to the search terms. After removing replicates and screening of titles and abstracts, 13 articles remained for further detailed evaluation (Fig. 1). Finally, a total of seven case-control studies were included in our meta-analysis, involving 1,074 IA cases and 1,500 control subjects. The main study characteristics are presented in Table I (19-23). Three studies involving 390 cases and 432 control subjects were from East Asian populations and four involved 684 cases and 1,068 control participants from Caucasian populations. In terms of the source of controls, threes studies included population-based studies, and four studies included hospital-based studies. Four studies were conducted in large samples and three in small samples. The genotype distributions among the controls of all studies were consistent with HWE except one study by Yu et al (23).

Meta-analysis. Table II summarizes the key results of the meta-analysis. In the overall analysis, a significant strong association between the ACE polymorphism and an increased risk of IA was identified in the allele (I vs. D; OR=1.27, 95\% CI=1.13-1.42; $\left.P_{O R}=0.000\right)$, homozygote comparison of codominant (II vs. DD; $\mathrm{OR}=1.64,95 \% \mathrm{CI}=1.30-2.07$; $\left.P_{O R}=0.000\right)$ and dominant (II+ID vs. DD; OR=1.38, 95\% $\left.\mathrm{CI}=1.05-1.80 ; P_{O R}=0.021\right)$ models. By contrast, no statistically significant association was detected under the heterozygote comparison of the codominant model (ID vs. DD; OR=1.25, 95\% CI=0.79-1.96; $P_{O R}=0.343$ ) and the recessive model (II vs. $\left.\mathrm{ID}+\mathrm{DD} ; \mathrm{OR}=1.35,95 \% \mathrm{CI}=0.98-1.87 ; P_{O R}=0.064\right)$.

When stratified by ethnicity, the ACE polymorphism showed a significant contribution to IA risk in the Asian population in all genetic models except in the recessive model (II vs. ID+DD; $\mathrm{OR}=1.15,95 \% \mathrm{CI}=0.71-1.87 ; P_{O R}=0.561$ ). In addition, a significant strong association was detected under the allele model (I vs. D; OR=1.21, 95\% CI=1.06-1.40; $P_{O R}=0.006$ ) and homozygote comparison of codominant model (II vs. DD; $\left.\mathrm{OR}=1.44,95 \% \mathrm{CI}=1.09-1.90 ; P_{O R}=0.011\right)$ in the Caucasian population (Table II; Fig. 2A-D).

In the subgroup analysis for the HB group, a significantly increased risk in all genetic models was observed except for in the recessive model (II vs. ID+DD; OR=1.15, 95\% CI=0.83-1.59; $\left.P_{O R}=0.401\right)$. Similar findings were revealed in the allele model ( $\mathrm{I}$ vs. $\mathrm{D}$; $\mathrm{OR}=1.28,95 \% \mathrm{CI}=1.08-1.52$; $\left.P_{O R}=0.004\right)$ and homozygote comparison of codominant model (II vs. DD; OR=1.56, 95\% CI=1.12-2.17; $P_{O R}=0.009$ ) in the PB subgroup (Table II).

When stratified by sample size, a statistically significant association was found in the large group in all of the genetic models. However, only the allele and homozygote comparison of codominant models showed significant associations in the small subgroup $\left(\mathrm{OR}=1.33,95 \% \mathrm{CI}=1.05-1.68 ; P_{O R}=0.019\right.$ and $\mathrm{OR}=1.93,95 \% \mathrm{CI}=1.19-3.13 ; P_{O R}=0.007$, respectively) (Table II).

Heterogeneity analysis. Significant heterogeneity in the ACE polymorphism was observed in the heterozygote comparison 


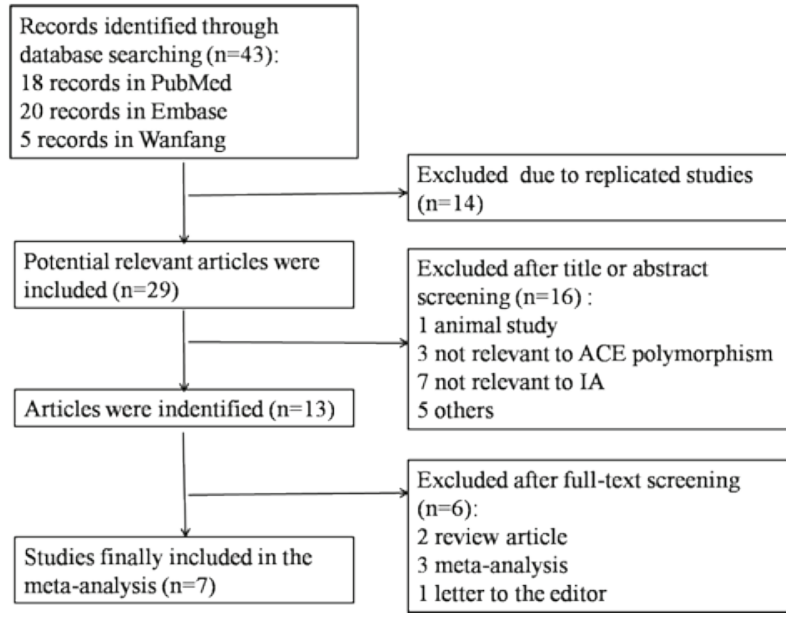

Figure 1. Flow chart presenting the detailed study selection process.

of codominant model $\left(\mathrm{I}^{2}=76.7 \% ; P_{h}=0.000\right)$, dominant model $\left(\mathrm{I}^{2}=44.5 \% ; P_{h}=0.094\right)$ and recessive model $\left(\mathrm{I}^{2}=66.1 \%\right.$; $\left.P_{h}=0.007\right)$. To investigate the potential sources of heterogeneity across studies, the pooled ORs under all comparisons were assessed via subgroup and sensitivity analyses. In the subgroup analysis by ethnicity, the heterogeneity of ACE was significant in the Caucasian studies in the heterozygote comparison of the codominant and recessive models $\left(\mathrm{I}^{2}=80.2 \% ; P_{h}=0.002\right.$ and $\mathrm{I}^{2}=74.9 \% ; P_{h}=0.008$, respectively). When stratified by source of control, the heterogeneity of ACE in the population-based studies was significant in the heterozygote comparison of codominant and recessive models $\left(\mathrm{I}^{2}=86.5 \%\right.$ and $P_{h}=0.001$; $\mathrm{I}^{2}=79 \%$ and $P_{h}=0.009$, respectively). When stratified by sample size, the heterogeneity of ACE in the small subgroup studies was significant in the heterozygote comparison of the codominant, dominant and recessive models $\left(\mathrm{I}^{2}=91.4 \%\right.$ and $P_{h}=0.000 ; \mathrm{I}^{2}=74 \%$ and $P_{h}=0.021 ;$ and $\mathrm{I}^{2}=86.9 \%$ and $P_{h}=0.000$, respectively) (Table II).

Notably, when the Caucasian population-based control study with $<300$ cases by Slowik et al (20) was excluded, the heterogeneity significantly decreased in the heterozygote comparison of codominant and recessive models (Table III). Therefore, the study by Slowik et al (20) may have contributed to the substantial heterogeneity of the ACE polymorphism.

Sensitivity analyses. Sensitivity analyses were performed by sequential deletion of single studies for all subjects to determine the influence of its individual data sets to the pooled OR, and the corresponding pooled ORs were not materially altered in the allele and homozygote comparison of the codominant models. The pooled ORs ranged from 1.11 to 1.42 in the heterozygote comparison of codominant model, 1.27 to 1.47 in the dominant model, and 1.21 to 1.48 in the recessive model (Table III). When excluding the study by Slowik et al (20), a significantly increased risk was identified with the ACE polymorphism in the heterozygous comparison of the codominant $\left(\mathrm{OR}=1.42,95 \% \mathrm{CI}=1.15-1.76 ; P_{O R}=0.001\right)$ and recessive $\left(\mathrm{OR}=1.21,95 \% \mathrm{CI}=1.00-1.47 ; P_{O R}=0.046\right)$ models (Table III; Fig. 3A and B). In addition, a significantly increased risk was detected after excluding the study by $\mathrm{Yu}$ et al (23) in the recessive model $\left(\mathrm{OR}=1.48,95 \% \mathrm{CI}=1.08-2.03 ; P_{O R}=0.01\right)$ 


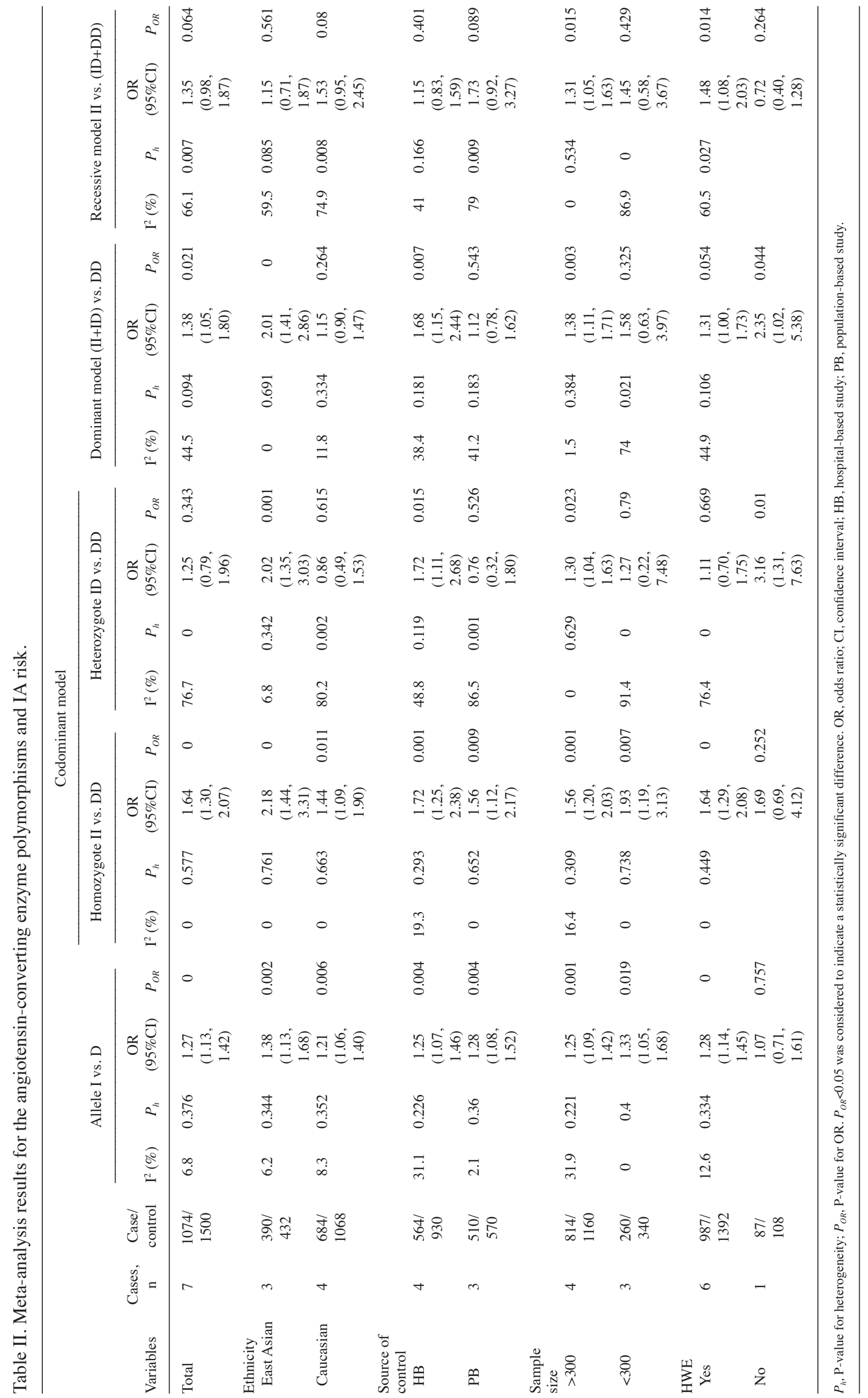


A

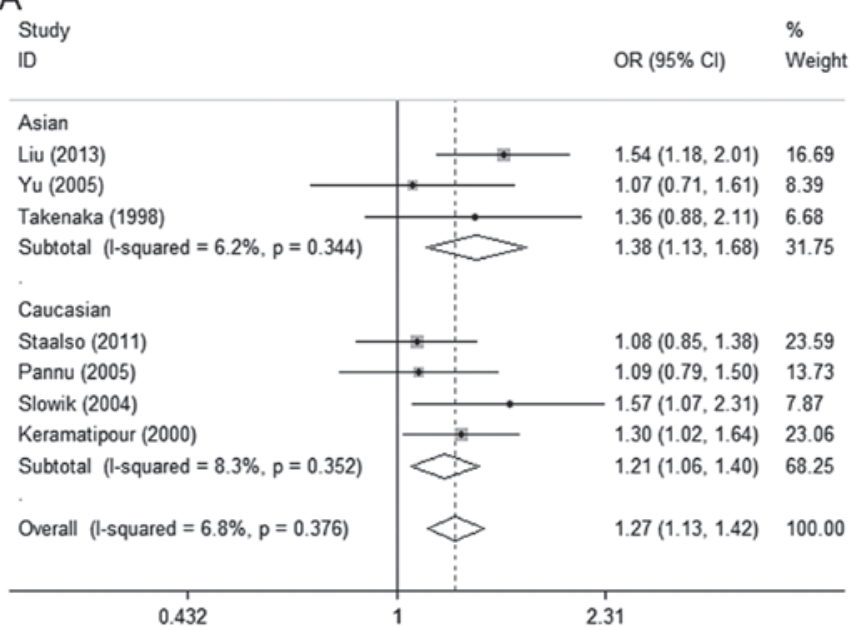

B

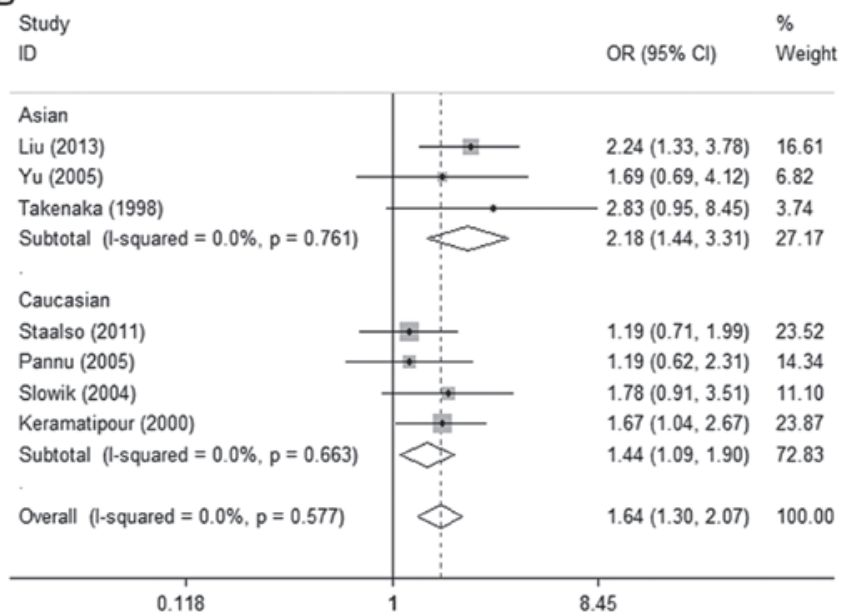

C

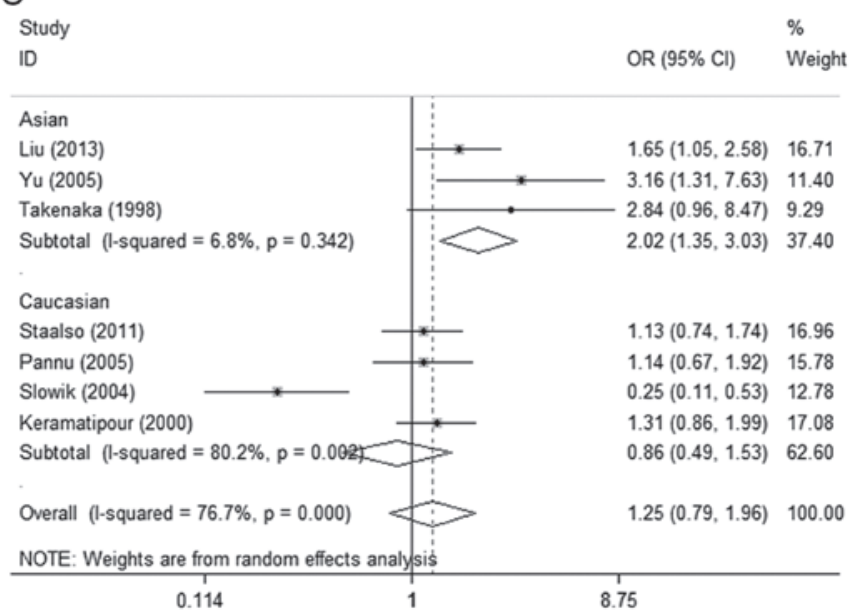

Figure 2. Forest plot of the association between the angiotensin-converting enzyme polymorphism and intracranial aneurysm susceptibility stratified by ethnicity. (A) Allele model (I vs. D); (B) homozygote comparison of codominant model (II vs. DD); (C) heterozygote comparison of codominant model (ID vs. DD).

(Table III and Fig. 3B). Furthermore, a significantly decreased risk was observed after excluding the study by Keramatipour et al (21) in the dominant model (OR=1.38, 95\% CI=0.98-1.94; $P_{O R}=0.067$ ) (Table III and Fig. 3C). Thus,
D

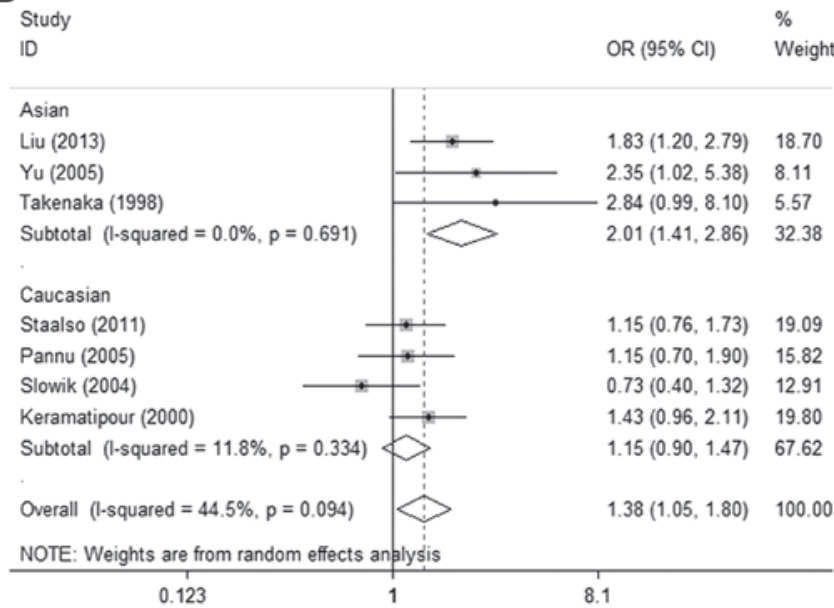

Figure 2. Continued. (D) Dominant model (II+ID vs. DD). CI, confidence interval; OR, odds ratio.

the overall association between the ACE polymorphism and IA risk was significantly influenced by these three studies.

Publication bias. Begg's funnel plot and Egger's test were performed to access the potential publication bias of the literature. The funnel plot shapes of these polymorphisms were symmetrical in each genetic model (Fig. 4 for the allele model). Subsequently, the Egger's test was used to provide statistical evidence of funnel plot symmetry. The results indicated that the current meta-analysis demonstrated a lack of publication bias in all of the genetic models (I vs. D: P=0.865; II vs. DD: $\mathrm{P}=0.542$; ID vs. $\mathrm{DD}$ : $\mathrm{P}=0.945$; II+ID vs. $\mathrm{DD}: \mathrm{P}=0.520$; II vs. ID+DD: $\mathrm{P}=0.956$. data not shown). The calculation results are consistent with the shape of the Begg's funnel plot.

\section{Discussion}

IA is a growing problem and leads to devastating consequences, such as SAH, hemiplegia and epilepsy. Rupture of IA is the most common cause of $\mathrm{SAH}$, which is associated with particularly high mortality and morbidity rates (3). Thus, it is important to identify the characteristics of individuals who are at high risk of IA and focus on regularly screening for IA. Previous studies indicate that genetics contribute to the development of IA (27-29). Furthermore, it is of great value to investigate the genetic architecture and establish the underlying molecular mechanism of IA. Until now, various polymorphisms in candidate genes have been considered as an important risk contributor to the development of IA.

Hypertension, which is a polygenic and multifactorial disorder, has been demonstrated to be a risk factor for IA (30). The RAAS plays a critical role in the development of hypertension and CVD (31). ACE is a key circulating enzyme in the RAAS, which catalyzes the conversion of angiotensin I to angiotensin II, and further degrades bradykinin (14). The D allele of the I/D polymorphism of a 287-bp Alu sequence within intron 16 of the $A C E$ gene has been reported to be correlated with increased circulating ACE levels in humans $(15,16)$. Furthermore, the ACE I/D polymorphisms 


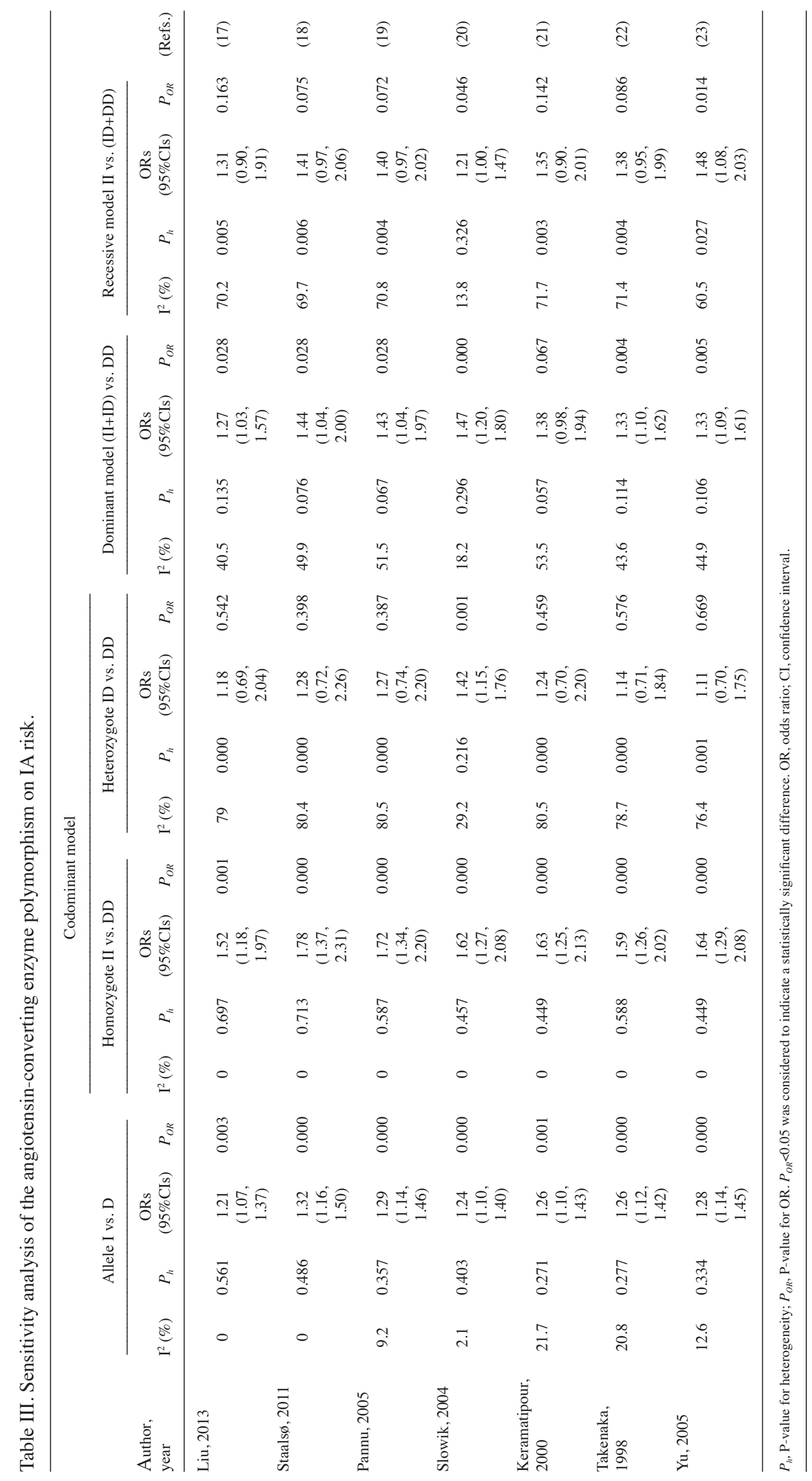



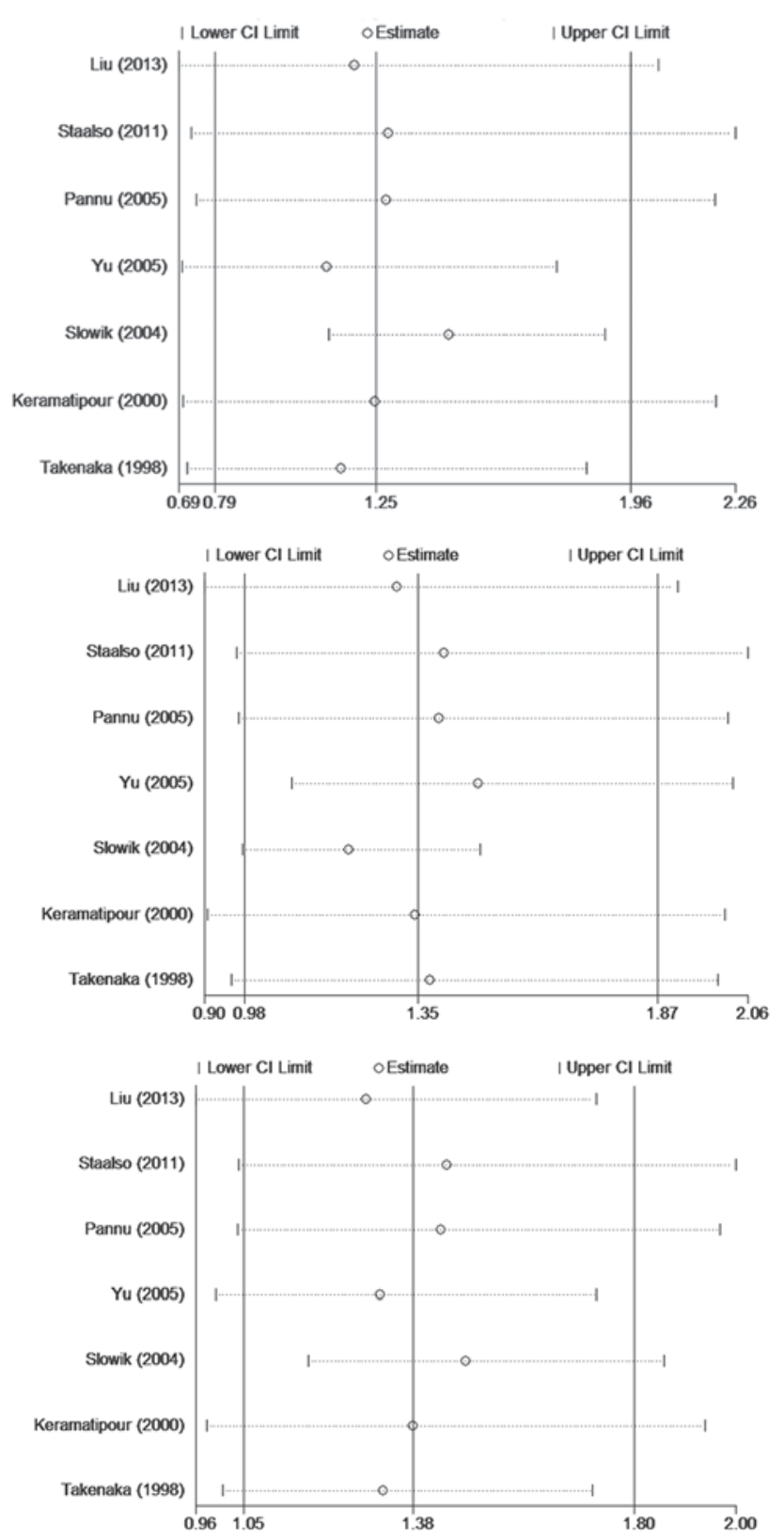

Figure 3. Sensitivity analysis of the angiotensin-converting enzyme polymorphism. Pooled odds ratio and 95\% CI of the six remaining studies, subsequent to exclusion of each individual study. (A) Heterozygote comparison of codominant model (ID vs. DD); (B) Recessive model (II vs. ID+DD); (C) Dominant model (II+ID vs. DD). CI, confidence interval.

have been extensively evaluated in numerous types of vascular disease (32). Therefore, ACE may present as a candidate gene for IA development.

A meta-analysis of two case-control studies by Keramatipour et al (21) showed that the I allele of the $A C E$ gene maybe a risk factor for IA. Subsequently, various studies with conflicting opinions have been reported $(18,19,23)$. A previous meta-analysis failed to detect an association between the ACE I/D polymorphism and IA susceptibility in the dominant $\left(\mathrm{OR}=1.23,95 \% \mathrm{CI}=0.82-1.85 ; P_{O R}=0.31\right)$ or the recessive $\left(\mathrm{OR}=1.58,95 \% \mathrm{CI}=0.98-2.57 ; P_{O R}=0.06\right)$ models (11). By contrast, another meta-analysis demonstrated a close association between the ACE I/D polymorphism and IA risk. In the current study, a comprehensive meta-analysis was conducted

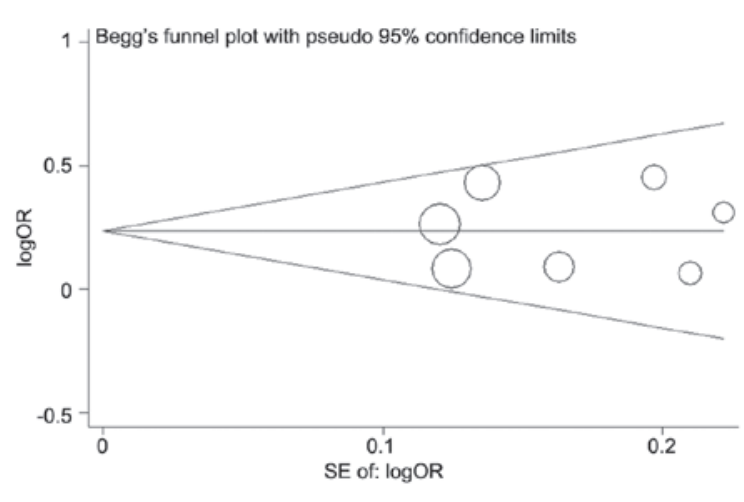

Figure 4. Begg's funnel plot to assess the publication bias of the angiotensin-converting enzyme polymorphism under the allele model (I vs. D). Each point corresponds to a separate study. OR, odds ratio.

with a markedly larger sample size to derive a more precise estimation of the association.

By combining data from seven case-control studies, including 1,074 IA patients and 1,500 control subjects, the present meta-analysis evaluated the association between the I/D polymorphisms of the $A C E$ gene and IA susceptibility. The pooled result showed a significant association between the I allele of the $A C E$ gene and IA risk $(\mathrm{OR}=1.27$, 95\% CI=1.13-1.42; $\left.P_{\mathrm{OR}}=0.000\right)$. The II genotype was associated with an increased risk for IA when compared with the DD genotype $\left(\mathrm{OR}=1.64,95 \% \mathrm{CI}=1.30-2.07 ; P_{O R}=0.000\right)$. The ACEII + ID genotype was correlated with a significantly increased risk for $\mathrm{IA}\left(\mathrm{OR}=1.38,95 \% \mathrm{CI}=1.05-1.80 ; P_{O R}=0.021\right)$.

In the subgroup analysis by ethnicity, an increased risk between ACE and IA was identified among Asian individuals (ID vs. DD: $\mathrm{OR}=2.02,95 \% \mathrm{CI}=1.35-3.03, P_{O R}=0.001$; II+ID vs. DD: $\left.\mathrm{OR}=2.01,95 \% \mathrm{CI}=1.41-2.86, P_{O R}=0.000\right)$, although not in Caucasian individuals (ID vs. DD: $\mathrm{OR}=0.86,95 \% \mathrm{CI}=0.49-1.53$, $P_{O R}=0.615$; II+ID vs. DD: $\mathrm{OR}=1.15,95 \% \mathrm{CI}=0.90-1.47$, $\left.P_{O R}=0.264\right)$. A number of factors may also be involved in the underlying mechanism for this difference between ethnic groups. In addition to the genetic backgrounds and the living environments, other factors, such as selection bias and different matching criteria may account for the different genetic effects. Thus, additional studies using different populations are warranted to further validate ethnic differences on the impact of the ACE polymorphism on IA risk.

Subgroup analysis by source of controls revealed a significantly increased risk among studies using hospital-based controls, but not population-based controls in the heterozygote comparison of codominant model and the dominant model, suggesting that the controls in the hospital-based studies may be sufficient to represent the general population. Thus, the use of proper and representative cancer-free control subjects is important in reducing bias in such genotype association studies.

Subgroup analysis by sample size revealed a significantly increased risk among studies using large samples in each genetic model, although no significance was found in the small sample subgroup in the heterozygote comparison of codominant, dominant and recessive models, suggesting that studies with large samples are required.

The statistical significance of genotype distributions was also detected in male and female groups (males: $\mathrm{OR}=3.56$, 
95\% CI=1.43-8.86; $P_{O R}=0.0006 ;$ females: OR=3.86, $\left.95 \% \mathrm{CI}=1.75-8.51 ; P_{O R}=0.0005\right)$ in the study by Slowik et al $(20)$. In addition, Liu et al (17) reported that no statistically significant differences were identified between genotypes in patients with IA, when stratified by the site, shape, size and Fisher Grade of aneurysms (17). In future studies, greater focus on clinical characteristics, such as gender, alcohol consumption, smoking, family history, and site, shape, size and Fisher Grade of IA should be taken into consideration to provide a more powerful analytical framework.

There were certain limitations of the current meta-analysis. First, the number of eligible studies and subjects of studies were relatively small, particularly for the subgroup analyses, which may result in insufficient power to detect a slight, although real effect of the ACE polymorphisms on IA risk. In addition, the study by $\mathrm{Yu}$ et al (23) whose genotype distribution in the control group was not consistent with HWE may contribute to the bias of the meta-analysis, as the results were affected after excluding this study in the sensitivity analysis. Finally, the results were based on single-factor estimates without adjustments for other risk factors.

In conclusion, this meta-analysis identified that the ACE polymorphism is associated with an increased risk of IA. However, large, well-designed multicenter studies are required to verify the present findings. In addition, further evaluation of the ACE polymorphism on IA risk should focus on the effect of gene-to-gene and gene-to-environment interactions.

\section{Acknowledgements}

The present study was supported by the National Science Foundation of China (grant nos. 81571141 and 81401802).

\section{References}

1. Rinkel GJ, Djibuti M, Algra A and van Gijn J: Prevalence and risk of rupture of intracranial aneurysms: A systematic review. Stroke 29: 251-256, 1998.

2. Korja M and Kaprio J: Controversies in epidemiology of intracranial aneurysms and SAH. Nat Rev Neurol 12: 50-55, 2016.

3. Hop JW, Rinkel GJ, Algra A and van Gijn J: Case-fatality rates and functional outcome after subarachnoid hemorrhage: A systematic review. Stroke 28: 660-664, 1997.

4. Yan J, Hitomi T, Takenaka K, Kato M, Kobayashi H, Okuda H, Harada KH and Koizumi A: Genetic study of intracranial aneurysms. Stroke 46: 620-626, 2015.

5. Tromp G, Weinsheimer S, Ronkainen A and Kuivaniemi H: Molecular basis and genetic predisposition to intracranial aneurysm. Ann Med 46: 597-606, 2014.

6. Rinkel GJ: Intracranial aneurysm screening: Indications and advice for practice. Lancet Neurol 4: 122-128, 2005.

7. Schievink WI: Genetics of intracranial aneurysms. Neurosurgery 40: 651-663, 1997.

8. Ronkainen A, Hernesniemi J, Puranen M, Niemitukia L, Vanninen R, Ryynänen M, Kuivaniemi H and Tromp G: Familial intracranial aneurysms. Lancet 349: 380-384, 1997.

9. Akiyama K, Narita A, Nakaoka H, Cui T, Takahashi T, Yasuno K, Tajima A, Krischek B, Yamamoto K, Kasuya H, et al: Genome-wide association study to identify genetic variants present in Japanese patients harboring intracranial aneurysms. J Hum Genet 55: 656-661, 2010.

10. Marchese E, Vignati A, Albanese A, Nucci CG, Sabatino G, Tirpakova B, Lofrese G, Zelano G and Maira G: Comparative evaluation of genome-wide gene expression profiles in ruptured and unruptured human intracranial aneurysms. J Biol Regul Homeost Agents 24: 185-195, 2010.
11. McColgan P, Thant KZ and Sharma P: The genetics of sporadic ruptured and unruptured intracranial aneurysms: A genetic meta-analysis of 8 genes and 13 polymorphisms in approximately 20,000 individuals. J Neurosurg 112: 714-721, 2010.

12. Ruigrok YM and Rinkel GJ: From GWAS to the clinic: Risk factors for intracranial aneurysms. Genome Med 2: 61, 2010.

13. Mohan D, Munteanu V, Coman T and Ciurea AV: Genetic factors involves in intracranial aneurysms-actualities. J Med Life 8: 336-341, 2015.

14. Skeggs LT, Dorer FE, Kahn JR, Lentz KE and Levine M: The biochemistry of the renin-angiotensin system and its role in hypertension. Am J Med 60: 737-748, 1976.

15. Rigat B, Hubert C, Alhenc-Gelas F, Cambien F, Corvol P and Soubrier F: An insertion/deletion polymorphism in the angiotensin I-converting enzyme gene accounting for half the variance of serum enzyme levels. J Clin Invest 86: 1343-1346, 1990.

16. Tiret L, Rigat B, Visvikis S, Breda C, Corvol P, Cambien F and Soubrier F: Evidence, from combined segregation and linkage analysis, that a variant of the angiotensin I-converting enzyme (ACE) gene controls plasma ACE levels. Am J Hum Genet 51: 197-205, 1992.

17. Liu Y, Li P, Hu X, Hu Y, Sun HG, Ma WC, Qiao F, He M and You C: Angiotensin-converting enzyme insertion/deletion gene polymorphism and risk of intracranial aneurysm in a Chinese population. J Int Med Res 41: 1079-1087, 2013.

18. Staals $\varnothing$ JM, Nielsen M, Edsen T, Koefoed P, Springborg JB, Moltke FB, Laursen H, Nielsen HB and Olsen NV: Common variants of the ACE gene and aneurysmal subarachnoid hemorrhage in a Danish population: A case-control study. J Neurosurg Anesthesiol 23: 304-309, 2011.

19. Pannu H, Kim DH, Seaman CR, Van Ginhoven G, Shete $S$ and Milewicz DM: Lack of an association between the angiotensin-converting enzyme insertion/deletion polymorphism and intracranial aneurysms in a Caucasian population in the United States. J Neurosurg 103: 92-96, 2005.

20. Slowik A, Borratynska A, Pera J, Betlej M, Dziedzic T, Krzyszkowski T, Czepko R, Figlewicz DA and Szczudlik A: II genotype of the angiotensin-converting enzyme gene increases the risk for subarachnoid hemorrhage from ruptured aneurysm. Stroke 35: 1594-1597, 2004.

21. Keramatipour M, McConnell RS, Kirkpatrick P, Tebbs S, Furlong RA and Rubinsztein DC: The ACE I allele is associated with increased risk for ruptured intracranial aneurysms. J Med Genet 37: 498-500, 2000

22. Takenaka K, Yamakawa H, Sakai H, Yoshimura S, Murase S, Okumura A, Nakatani K, Kimura T, Nishimura Y, Yoshimi N and Sakai N: Angiotensin I-converting enzyme gene polymorphism in intracranial saccular aneurysm individuals. Neurol Res 20: 607-611, 1998.

23. Yu S, Zhao J, Zhang D, Bi Z and Kang S: A study of the relationship between polymorphism of the angiotensin converting enzyme gene insertion/delection and intracranial aneurysm. J Cap Univ Med Sci 4: 380-382, 2005.

24. Mantel N and Haenszel W: Statistical aspects of the analysis of data from retrospective studies of disease. J Natl Cancer Inst 22: 719-748, 1959.

25. DerSimonian R and Kacker R: Random-effects model for meta-analysis of clinical trials: An update. Contemp Clin Trials 28: 105-114, 2007.

26. Egger M, Davey Smith G, Schneider M and Minder C: Bias in meta-analysis detected by a simple, graphical test. BMJ 315: 629-634, 1997.

27. Kojima M, Nagasawa S, Lee YE, Takeichi Y, Tsuda E and Mabuchi N: Asymptomatic familial cerebral aneurysms. Neurosurgery 43: 776-781, 1998.

28. Nakagawa T and Hashi K: The incidence and treatment of asymptomatic, unruptured cerebral aneurysms. J Neurosurg 80: 217-223, 1994.

29. Caranci F, Briganti F, Cirillo L, Leonardi M and Muto M: Epidemiology and genetics of intracranial aneurysms. Eur J Radiol 82: 1598-1605, 2013

30. Inci S and Spetzler RF: Intracranial aneurysms and arterial hypertension: A review and hypothesis. Surg Neurol 53: 530-542, 2000.

31. Lavoie JL and Sigmund CD: Minireview: Overview of the renin-angiotensin system-an endocrine and paracrine system. Endocrinology 144: 2179-2183, 2003.

32. Sayed-Tabatabaei FA, Oostra BA, Isaacs A, van Duijn CM and Witteman JC: ACE polymorphisms. Circ Res 98: 1123-1133, 2006. 